IJMMS 26:1 (2001) 35-44

PII. S0161171201003581

http://ijmms.hindawi.com

(C) Hindawi Publishing Corp.

\title{
ASYMPTOTIC BEHAVIOUR OF SOLUTIONS FOR POROUS MEDIUM EQUATION WITH PERIODIC ABSORPTION
}

\author{
YIN JINGXUE and WANG YIFU
}

(Received 28 June 1999)

\begin{abstract}
This paper is concerned with porous medium equation with periodic absorption. We are interested in the discussion of asymptotic behaviour of solutions of the first boundary value problem for the equation. In contrast to the equation without sources, we show that the solutions may not decay but may be "attracted" into any small neighborhood of the set of all nontrivial periodic solutions, as time tends to infinity. As a direct consequence, the null periodic solution is "unstable." We have presented an accurate condition on the sources for solutions to have such a property. Whereas in other cases of the sources, the solutions might decay with power speed, which implies that the null periodic solution is "stable."
\end{abstract}

2000 Mathematics Subject Classification. 35K35, 35K57.

1. Introduction. This paper is concerned with the following nonlinear diffusion equation with periodic absorption

$$
\begin{gathered}
\frac{\partial u}{\partial t}=\Delta u^{m}+a(x, t) u^{p} \quad \text { in } \Omega \times(0,+\infty), \\
u(x, t)=0 \quad \text { on } \partial \Omega \times(0,+\infty), \\
u(x, 0)=u_{0}(x) \quad \text { in } \Omega,
\end{gathered}
$$

where $m>1, \Omega$ is a bounded domain in $\mathbb{R}^{N}$ with smooth boundary $\partial \Omega, a(x, t)$ is smooth, strictly positive and periodic in time with period $\omega>0$, and $u_{0}(x)$ is smooth and nonnegative.

Equations of the form (1.1) have been suggested as mathematical models of several natural phenomena, such as reaction-diffusion processes, population dynamics, etc., (cf. $[1,2,3,5,6,9,11]$ and the references therein). For the equation with $a(x, t)$ independent of $t$, such an equation has been deeply investigated. In particular, if $1 \leq p<m$, it has been shown in [3,11,12] that, for $1 \leq p<m$, all solutions of the initial boundary problem (1.1), (1.2), and (1.3) converge to unique positive steady states solutions as time tends to infinity.

There are many diffusion processes affected by periodic absorption, such as season, generation, and so on. This motivates us to investigate the equation with periodic absorption. However, for the equation with periodic absorption, it is of no meaning to consider the steady states. So, it is much interesting to find a new way to describe the asymptotic behaviour of solutions of the initial boundary value problem. The purpose of this paper is devoted to the discussion to such a problem. We will show that if $1 \leq p<m$, then there exists an attractor which consists of all nontrivial periodic 
solutions. Precisely speaking, any nontrivial solution of the initial boundary value problem (1.1), (1.2), and (1.3) will be attracted into any small neighbourhood of the attractor as time tends to infinity. As an immediate consequence, the null periodic solution is "unstable." However, such a property might not be valid for other cases of sources. We show that if $p=m$, then the null periodic solution attracts all solutions of the initial boundary value problem, provided that $a(x, t)<\lambda_{1}$, where $\lambda_{1}$ is the first eigenvalue of Laplacian with zero Dirichlet boundary value condition. In such a case, the null periodic solution is "stable." On the other hand, if $a(x, t)>\lambda_{1}$, then the solution of the initial boundary value problem might blow up at finite or infinite time. Moreover, if $1<p / m<(N+2) /(N-2)$, then the null periodic solution is "stable" too. In this case, the null periodic solution attracts solutions with "small initial data." However, it will not attract solutions with "large initial data."

This paper is constructed as follows. As preliminaries, in Section 2, we introduce the weak formulation of solutions and state the main results. Section 3 is devoted to the proof of the existence of periodic solutions for the problem (1.1), (1.2) by using the monotone iteration technique, which is different from that adopted in $[7,8]$. Subsequently, we present the proof of the main results in Section 4.

2. Preliminaries and the main results. Let $T>0$ and set $Q_{T}=\Omega \times(0, T)$. Because $m>1$, equation (1.1) is of degenerate type, and so we could not expect to find classical solutions. This leads to the following weak formulation of solutions.

DEFINITION 2.1. A nonnegative and continuous function $u$ is called a solution to the problem (1.1), (1.2), and (1.3), if

(i) For any $T>0, u$ satisfies the condition (1.2) in the usual sense and $\left|\nabla u^{m}\right| \in$ $L^{2}\left(Q_{T}\right)$.

(ii) For any $T>0$, the following integral equality holds:

$$
\begin{aligned}
& \iint_{Q_{T}}(\left.u \frac{\partial \varphi}{\partial t}-\nabla u^{m} \nabla \varphi+a(x, t) u^{p} \varphi\right) d x d t \\
& \quad=\int_{\Omega} \varphi(x, 0) u_{0}(x) d x-\int_{\Omega} u(x, T) \varphi(x, T) d x
\end{aligned}
$$

for all $\varphi \in C^{1}\left(\bar{Q}_{T}\right)$ with $\varphi(x, t)=0$ for $(x, t) \in \partial \Omega \times(0, T)$.

If "=" is replaced by " $\leq$ " $(\geq)$ in the above equality with an additional assumption $\varphi(x, t) \geq 0$, then $u$ is said to be a supersolution (subsolution) to problem (1.1), (1.2), and (1.3).

DEFINITION 2.2. A continuous function $u$ is said to be a periodic solution of problem (1.1), (1.2), if it is a solution of (1.1), (1.2) such that $u \in C_{\omega}(\bar{Q})$. Here $Q=\Omega \times(0, \infty)$ and $C_{\omega}(\bar{Q})$ denotes the set of all the continuous functions with time period $\omega$.

Now, we state the main results of this paper.

THeOREM 2.3. Assume that $1 \leq p<m$. Then problem (1.1), (1.2) has a minimal and a maximal nonnegative nontrivial periodic solutions $u_{*}(x, t)$ and $u^{*}(x, t)$. Moreover, if $u(x, t)$ is the solution of the initial boundary value problem (1.1), (1.2), and (1.3) with 
$u_{0}(x)>0$ for $x \in \Omega$, then for any $\varepsilon>0$,

$$
u_{*}(x, t)-\varepsilon \leq u(x, t) \leq u^{*}(x, t)+\varepsilon
$$

holds for $x \in \Omega$ and sufficiently large $t$.

REMARK 2.4. Theorem 2.3 implies that the null periodic solution is "unstable."

THEOREM 2.5. Assume that $p=m$ and $a(x, t)<\lambda_{1}$, where $\lambda_{1}$ is the first eigenvalue of the Laplacian with zero Dirichlet boundary value condition. Then any solution of problem (1.1), (1.2), and (1.3) decays to zero powerly as t tends to infinity.

THEOREM 2.6. Assume that $p=m$ and $a(x, t)>\lambda_{1}$. Then the solution of problem (1.1), (1.2), and (1.3) might blow up at finite or infinite time.

THEOREM 2.7. Assume that $1<p / m \leq(N+2) /(N-2)$. If the initial data is "small" enough, then the solution of problem (1.1), (1.2), and (1.3) decays to zero powerly as $t$ tends to infinity.

REMARK 2.8. The results in Theorems 2.5 and 2.7 imply that the null periodic solution is "stable." However, it will not attract solutions with "large initial data."

3. Existence of periodic solutions. We first state two lemmas, which will be used in our arguments.

LEMMA 3.1 (comparison [2]). Let $\underline{u}, \bar{u}$ be the subsolution and supersolution of problem (1.1), (1.2), and (1.3) with initial value $\underline{u}_{0}(x), \bar{u}_{0}(x)$, respectively. Then $\underline{u}(x, t)$ $\leq \bar{u}(x, t)$, provided that $\underline{u}_{0} \leq \bar{u}_{0}$.

LEMMA 3.2 (regularity [4, 10]). Let $u$ be the solution of the equation

$$
\frac{\partial u}{\partial t}=\Delta u^{m}+f(x, t), \quad(m>1)
$$

subject to the homogeneous Dirichlet condition (1.2). If $f \in L^{\infty}\left(Q_{T}\right)$, then there exist positive constants $K$ and $\alpha \in(0,1)$ depending only upon $\tau \in(0, T)$ and $\|f\|_{\infty}$ such that for any $\left(x_{i}, t_{i}\right) \in \bar{\Omega} \times[\tau, T](i=1,2)$,

$$
\left|u\left(x_{1}, t_{1}\right)-u\left(x_{2}, t_{2}\right)\right| \leq K\left(\left|x_{1}-x_{2}\right|^{\alpha}+\left|t_{1}-t_{2}\right|^{\alpha / 2}\right) .
$$

Proposition 3.3. Assume that $1 \leq p<m$. Then the problem (1.1), (1.2) has at least one nonnegative nontrivial periodic solution.

PROoF. Let $\lambda_{1}, \varphi_{1}$ be the first eigenvalue and its corresponding eigenfunction to the Laplacian operator $-\Delta$ on the domain $\Omega, \mu_{1}, \psi_{1}$ be the first eigenvalue and its corresponding eigenfunction to the Laplacian operator $-\Delta$ on some domain $\Omega^{\prime} \ni \Omega$, with respect to homogeneous Dirichlet data, respectively. It is clear that $\psi_{1}(x)>0$ for all $x \in \bar{\Omega}$. Denoted by

$$
a_{L}=\min _{\bar{\Omega} \times[0, \omega]} a(x, t), \quad a_{M}=\max _{\bar{\Omega} \times[0, \omega]} a(x, t),
$$

and define

$$
\underline{u}=\left(\rho \varphi_{1}\right)^{1 / m}, \quad \bar{u}=\left(R \psi_{1}\right)^{1 / m},
$$


where

$$
\rho=\frac{\left(a_{L} / \lambda_{1}\right)^{m /(m-p)}}{\max _{\bar{\Omega}} \varphi_{1}}, \quad R=\frac{\left(a_{M} / \mu_{1}\right)^{m /(m-p)}}{\min _{\bar{\Omega}} \psi_{1}} .
$$

Clearly, $\underline{u}$ and $\bar{u}$ are the subsolution and supersolution of (1.1) subject to the condition (1.2), respectively. Further, we may assume $\underline{u} \leq \bar{u}$, else we may change $\Omega^{\prime}$ and then $R, \rho$ appropriately.

Now, we define a Poincaré map $T: C(\bar{\Omega}) \rightarrow C(\bar{\Omega}), T\left(u_{0}(x)\right)=u(x, \omega)$, where $u(x, t)$ is the solution of problem (1.1), (1.2), and (1.3) with initial datum $u_{0}(x)$. By the results of $[2,11]$, the map $T$ is well defined.

Let $u_{n}(x, t)$ be the solution of problem (1.1), (1.2), and (1.3) with initial value $u_{0}(x)=$ $T^{n-1} \underline{u}(x)$. It is observed that $u_{n}(x, \omega)=T^{n} \underline{u}(x)$ and $u_{n}(x, t) \leq \bar{u}(x)$. Moreover, by a rather standard argument, we can conclude that there exist a function $v \in C(\bar{\Omega})$ and the subsequence of $\left\{T^{n} \underline{u}\right\}_{n=1}^{\infty}$, denoted by itself for simplicity, such that

$$
T^{n} \underline{u} \longrightarrow v \text { in } C(\bar{\Omega}) .
$$

Now we claim that the solution of problem (1.1), (1.2), and (1.3) with $u_{0}(x)=v(x)$ is a nonnegative nontrivial periodic solution.

To show this, by $u_{n}(x, t) \leq \bar{u}(x)$ and Lemma 3.2, we first get that for $\left(x_{i}, t_{i}\right) \in$ $\bar{\Omega} \times[\omega, 2 \omega]$

$$
\left|u_{n}\left(x_{1}, t_{1}\right)-u_{n}\left(x_{2}, t_{2}\right)\right| \leq K\left(\left|x_{1}-x_{2}\right|^{\alpha}+\left|t_{1}-t_{2}\right|^{\alpha / 2}\right),
$$

where positive constants $K$ and $\alpha$ are independent of $n$.

Next, it follows from [2] that there exists a constant $C$, which is independent of $n$, such that

$$
\max _{(\omega, 2 \omega)}\left\|\nabla u_{n}^{m}\right\|_{L^{2}(\Omega)} \leq C, \quad\left\|\left(u_{n}^{m}\right)_{t}\right\|_{L^{2}(\Omega \times(\omega, 2 \omega))} \leq C .
$$

Hence there exist a function $W(x, t) \in C(\bar{\Omega} \times[\omega, 2 \omega])$ and the subsequence of $\left\{u_{n}\right\}_{n=1}^{\infty}$, denoted by itself, such that

$$
\begin{gathered}
u_{n} \rightarrow W \quad \text { in } C(\bar{\Omega} \times[\omega, 2 \omega]), \\
\nabla u_{n}^{m} \rightarrow \nabla W^{m} \text { in } L^{2}(\Omega \times(\omega, 2 \omega)),
\end{gathered}
$$

and thus $v(x)=W(x, \omega)$. Moreover, $W(x, t)$ is the solution of (1.1) and (1.2) in the domain $\Omega \times(\omega, 2 \omega)$.

Finally, we conclude that $W(x, 2 \omega)=W(x, \omega)$. In fact

$$
\begin{aligned}
W(x, 2 \omega) & =\lim _{n \rightarrow \infty} u_{n}(x, 2 \omega)=\lim _{n \rightarrow \infty} T\left(u_{n}(x, \omega)\right)(x) \\
& =\lim _{n \rightarrow \infty} T\left(T\left(T^{n} \underline{u}\right)\right)(x)=\lim _{n \rightarrow \infty} T^{n+2} \underline{u}(x) \\
& =\lim _{n \rightarrow \infty} T^{n+1} \underline{u}(x)=\lim _{n \rightarrow \infty} T\left(T^{n} \underline{u}\right)(x) \\
& =\lim _{n \rightarrow \infty} u_{n}(x, \omega)=W(x, \omega) .
\end{aligned}
$$

Therefore, according to the uniqueness of the solution to problem (1.1), (1.2), and (1.3), we can conclude that $u(x, t)$, the solution of problem (1.1), (1.2), and (1.3) with $u(x, 0)=v(x)$, is indeed a nonnegative nontrivial periodic solution. This completes the proof of the proposition. 
4. Proof of the main results. We devote this section to the proof of the main results stated in Section 2. We begin with the proof of Theorem 2.3

Proof OF TheOrem 2.3. Let $\underline{u}(x, t)$ be the solution of the problem

$$
\begin{gathered}
u_{t}=\Delta u^{m}+a_{L} u^{p} \quad \text { in } \Omega \times \mathbb{R}, \\
u(x, t)=0 \quad \text { on } \partial \Omega \times \mathbb{R}, \\
u(x, 0)=u_{0}(x) \quad \text { in } \bar{\Omega},
\end{gathered}
$$

and $\bar{u}(x, t)$ be the solution to problem

$$
\begin{gathered}
u_{t}=\Delta u^{m}+a_{M} u^{p} \quad \text { in } \Omega \times \mathbb{R}, \\
u(x, t)=0 \quad \text { on } \partial \Omega \times \mathbb{R}, \\
u(x, 0)=u_{0}(x) \quad \text { in } \bar{\Omega},
\end{gathered}
$$

respectively. Then Sacks in [11] shows that there exist positive functions $\underline{v}(x)$ and $\bar{v}(x)$ such that

$$
\lim _{t \rightarrow \infty}\|\underline{u}(x, t)-\underline{v}(x)\|_{C(\bar{\Omega})}=0, \quad \lim _{t \rightarrow \infty}\|\bar{u}(x, t)-\bar{v}(x)\|_{C(\bar{\Omega})}=0,
$$

provided that $u_{0}(x)>0$ for $x \in \Omega$.

On the other hand, the comparison result in Lemma 3.1 yields

$$
\underline{u}(x, t) \leq u(x, t) \leq \bar{u}(x, t) .
$$

Just as in the proof of Proposition 3.3, we may choose $\Omega_{1} \Subset \Omega$, such that

$$
\underline{u}(x, t) \geq\left(\rho \varphi_{1}\right)^{1 / m}, \quad \bar{u}(x, t) \leq\left(R \psi_{1}\right)^{1 / m},
$$

where $\varphi_{1}$ and $\psi_{1}$ are the eigenfunctions corresponding to the first eigenvalues to the Laplacian operator $-\Delta$ on the domain $\Omega_{1}$ and $\Omega$, respectively. It follows that

$$
\left(\rho \phi_{1}\right)^{1 / m} \leq u(x, t) \leq\left(R \psi_{1}\right)^{1 / m} .
$$

We assume that $\underline{v}(x, t)$ is the solution of problem (1.1), (1.2), and (1.3) with initial datum $\left(\rho \phi_{1}\right)^{1 / m}, \bar{v}(x, t)$ is the solution of problem (1.1), (1.2), and (1.3) with initial datum $\left(R \psi_{1}\right)^{1 / m}$, respectively. Then from (4.6) we have

$$
\underline{v}\left(x, t+m \omega+T_{0}\right) \leq u\left(x, t+m \omega+T_{0}\right) \leq \bar{v}\left(x, t+m \omega+T_{0}\right)
$$

for $x \in \bar{\Omega}, t \in[0, \omega]$, and $m=0,1,2 \ldots$

If we define $\underline{v}_{m}(x, t)=\underline{v}\left(x, t+m \omega+T_{0}\right), \bar{v}_{m}(x, t)=\bar{v}\left(x, t+m \omega+T_{0}\right)$, and $u_{m}(x, t)$ $=u\left(x, t+m \omega+T_{0}\right)$, then (3.9) can be rewritten as

$$
\underline{v}_{m}(x, t) \leq u_{m}(x, t) \leq \bar{v}(x, t)
$$

for $(x, t) \in \bar{\Omega} \times[0, \omega]$. 
On the other hand, the argument as the one used in the proof of Proposition 3.3 shows that

$$
\lim _{m \rightarrow \infty} \underline{v}_{m}(x, t)=u_{*}(x, t), \quad \lim _{m \rightarrow \infty} \bar{v}_{m}(x, t)=u^{*}(x, t),
$$

here $u_{*}(x, t), u^{*}(x, t)$ are the minimal and maximal nonnegative nontrivial periodic solutions.

Therefore, for each $\varepsilon>0$, there exists $m_{0}$ such that $m \geq m_{0}$

$$
u_{*}(x, t)-\varepsilon \leq u_{m}(x, t) \leq u^{*}(x, t)+\varepsilon
$$

for $x \in \bar{\Omega}, t \in[0, \omega]$, provided that the periodicity of $u_{*}(x, t)$ and $u^{*}(x, t)$ is taken into account, and thus the proof of the theorem is completed.

REMARK 4.1. The approach can be applied to the reaction-diffusion system of the form

$$
\frac{\partial u_{i}}{\partial t}=\Delta u_{i}^{m_{i}}+b_{i}(x, t) u_{1}^{p_{i}} u_{2}^{q_{i}},
$$

where $m_{i}>1, \omega>0, p_{i}, q_{i} \geq 1, b_{i}(x, t)>0$,

$$
b_{i}(x, t+\omega)=b_{i}(x, t), \quad \frac{p_{i}}{m_{1}}+\frac{q_{i}}{m_{2}}<1 \quad(i=1,2) .
$$

The result similar to Theorem 2.3 can be obtained.

Proof OF TheOrem 2.5. Let $u$ be a solution of problem (1.1), (1.2), and (1.3). Without loss of generality, we may further assume that

$$
\alpha \equiv \int_{\Omega} u_{0}^{m+1}(x) d x>0 .
$$

Let $v=u^{m}$ and take $v$ as a test function, after taking an approximating procedure, we obtain

$$
\begin{aligned}
\frac{1}{m+1} \frac{d}{d t} \int_{\Omega} v^{1 / m+1} d x & =-\int_{\Omega}|\nabla v|^{2} d x+\int_{\Omega} a(x, t) v^{2} d x \\
& \leq-\lambda_{1} \int_{\Omega} v^{2} d x+a_{M} \int_{\Omega} v^{2} d x \\
& =-\left(\lambda_{1}-a_{M}\right) \int_{\Omega} v^{2} d x .
\end{aligned}
$$

The Hölder inequality then implies for some $\mu>0$ that

$$
\frac{d}{d t} \int_{\Omega} v^{(m+1) / m} d x \leq-\mu\left(\int_{\Omega} v^{(m+1) / m} d x\right)^{2 m /(m+1)} .
$$

Setting $\mu_{1}=\mu(m-1), \beta=2 m /(m+1), \mu_{2}=\alpha^{1-\beta}$, and integrating the above inequality, we have

$$
\int_{\Omega} v^{(m+1) / m} d x \leq \frac{1}{\left(\mu_{1} t+\mu_{2}\right)^{1 /(\beta-1)}} .
$$

This completes the proof. 
Proof OF TheOrem 2.6. Let $u$ be a solution of problem (1.1), (1.2), and (1.3) with initial datum $u_{0}(x) \geq \phi_{1}(x)^{1 / m}$, where $\phi_{1}$ is the eigenfunction of $-\Delta$ corresponding to the first eigenvalue $\lambda_{1}$ with zero Dirichlet boundary value condition. It is easily seen that $\phi_{1}(x)$ is a subsolution of problem (1.1), (1.2), and (1.3).

Taking $\phi_{1}(x)$ as a test function, we see that

$$
\begin{aligned}
\frac{d}{d t} \int_{\Omega} u \phi_{1} d x & =\int_{\Omega} u^{m}\left(\Delta \phi_{1}+a(x, t) \phi_{1}\right) d x \\
& =\int_{\Omega} u^{m}\left(-\lambda_{1} \phi_{1}+a(x, t) \phi_{1}\right) d x .
\end{aligned}
$$

Because $u_{0}(x) \geq \phi_{1}(x)^{1 / m}$, the comparison principle implies that $u(x, t) \geq \phi_{1}(x)^{1 / m}$. Therefore,

$$
\frac{d}{d t} \int_{\Omega} u \phi_{1} d x \geq\left(\sup a(x, t)-\lambda_{1}\right) \int_{\Omega} \phi_{1}^{2} d x,
$$

from which we immediately see that $u(x, t)$ must blow up at finite or infinite time. This completes the proof.

To prove Theorem 2.7, we need the following technical lemma.

LEMMA 4.2. Let $\beta>1, a>0$, and $f(s)=s-a s^{\beta}$. Then there exists a positive constant $\varepsilon_{0}$, such that for any $0<\varepsilon<\varepsilon_{0}$,

$$
f(2 \varepsilon)>\varepsilon
$$

Proof. Choosing

$$
0<\varepsilon<\frac{1}{2^{\beta /(\beta-1)} a^{1 /(\beta-1)}},
$$

we immediately obtain

$$
\begin{aligned}
f(2 \varepsilon) & =2 \varepsilon-a(2 \varepsilon)^{\beta}=2 \varepsilon\left(1-a(2 \varepsilon)^{\beta-1}\right) \\
& >2 \varepsilon\left[1-a 2^{\beta-1}\left(\frac{1}{2^{\beta /(\beta-1)} a^{1 /(\beta-1)}}\right)^{\beta-1}\right]=\varepsilon .
\end{aligned}
$$

This completes the proof.

Proof OF TheOrem 2.7. Let $u$ be a solution of problem (1.1), (1.2), and (1.3). Without loss of generality, we may further assume that

$$
\alpha \equiv \int_{\Omega} u_{0}^{m+1}(x) d x>0 .
$$

Just as in the proof of Theorem 2.5, we let $v=u^{m}$ and take $v$ as a test function and obtain

$$
\frac{1}{m+1} \frac{d}{d t} \int_{\Omega} v^{1 / m+1} d x=-\int_{\Omega}|\nabla v|^{2} d x+\int_{\Omega} a(x, t) v^{p / m+1} d x .
$$

The crucial step is to estimate the second integral in the above equality. For this purpose, we consider the auxiliary problem

$$
\begin{gathered}
\frac{\partial w^{1 / m}}{\partial t}=\Delta w+a_{M} w^{p / m} \quad \text { in } \Omega \times(0,+\infty), \\
w(x, t)=0 \quad \text { on } \partial \Omega \times(0,+\infty) \\
w(x, 0)=v_{0}(x) \quad \text { in } \Omega .
\end{gathered}
$$


Taking $\partial w / \partial t$ as a test function, we obtain

$$
\frac{1}{m} \int_{\Omega} w^{1 / m-1}\left(\frac{\partial w}{\partial t}\right)^{2} d x=-\frac{1}{2} \frac{d}{d t} \int_{\Omega}|\nabla w|^{2} d x+\frac{a_{M} m}{p+m} \frac{d}{d t} \int_{\Omega} w^{(p+m) / m} d x .
$$

Thus

$$
\begin{aligned}
& \frac{1}{2} \int_{\Omega}|\nabla w|^{2} d x-\frac{a_{M} m}{p+m} \int_{\Omega} w^{(p+m) / m} d x \\
& \quad \leq \frac{1}{2} \int_{\Omega}\left|\nabla w_{0}\right|^{2} d x-\frac{a_{M} m}{p+m} \int_{\Omega} w_{0}^{(p+m) / m} d x
\end{aligned}
$$

and hence

$$
\int_{\Omega}|\nabla w|^{2} d x \leq \int_{\Omega}\left|\nabla w_{0}\right|^{2} d x+\frac{2 a_{M} m}{p+m} \int_{\Omega} w^{(p+m) / m} d x .
$$

The Poincaré inequality then implies

$$
\int_{\Omega} w^{(p+m) / m} d x \leq C_{1}\left(\int_{\Omega}\left|\nabla w_{0}\right|^{2} d x\right)^{(p+m) / 2 m}+C_{2}\left(\int_{\Omega} w^{(p+m) / m} d x\right)^{(p+m) / 2 m},
$$

where $C_{1}$ and $C_{2}$ are constants depending only on $p, m$, and $a_{M}$. Setting $\beta=(p+$ $m) / 2 m$ and

$$
g(t)=\int_{\Omega} w(x, t)^{(p+m) / m} d x, \quad f(s)=s-C_{2} s^{\beta},
$$

we see that

$$
f(g(t)) \leq C_{1}\left\|\nabla w_{0}\right\|^{2 \beta} .
$$

By virtue of Lemma 4.2, there exists a constant $\varepsilon_{0}>0$, such that

$$
f(2 \varepsilon)>\varepsilon, \text { for any } \varepsilon<\varepsilon_{0} \text {. }
$$

Now, we assume that $0<\left\|\nabla w_{0}\right\|^{2 \beta}<\varepsilon_{0} / C_{1}$ and hence

$$
f\left(2 C_{1}\left\|\nabla w_{0}\right\|^{2 \beta}\right)>C_{1}\left\|\nabla w_{0}\right\|^{2 \beta} .
$$

In addition, we assume

$$
g(0) \equiv \int_{\Omega} w_{0}(x)^{(p+m) / m} d x
$$

is small enough, such that

$$
f(g(0))<C_{1}\left\|\nabla w_{0}\right\|^{2 \beta}
$$

It follows from (4.30) and the continuity of $g(t)$ that

$$
g(t) \leq 2 C_{1}\left\|\nabla w_{0}\right\|^{2 \beta},
$$

that is,

$$
\int_{\Omega} w(x, t)^{(p+m) / m} d x \leq 2 C_{1}\left(\int_{\Omega}\left|\nabla v_{0}(x)\right|^{2} d x\right)^{(p+m) / m} .
$$


Now, we turn to the estimates on $v$. First, we notice that the estimate (4.36) and the comparison technique imply that

$$
\int_{\Omega} v(x, t)^{(p+m) / m} d x \leq 2 C_{1}\left(\int_{\Omega}\left|\nabla v_{0}(x)\right|^{2} d x\right)^{(p+m) / m}
$$

By virtue of (4.23) and the Poincaré inequality, we see that

$$
\begin{aligned}
& \frac{1}{m+1} \frac{d}{d t} \int_{\Omega} v^{1 / m+1} d x \\
& \quad \leq-\int_{\Omega}|\nabla v|^{2} d x+a_{M} \int_{\Omega} v^{p / m+1} d x \\
& \quad=-\int_{\Omega}|\nabla v|^{2} d x+a_{M}\left(\int_{\Omega} v^{(p+m) / m} d x\right)^{2 m /(p+m)}\left(\int_{\Omega} v^{(p+m) / m} d x\right)^{(p-m) /(p+m)} \\
& \quad \leq-\int_{\Omega}|\nabla v|^{2} d x+C_{3} \int_{\Omega}|\nabla v|^{2} d x\left(\int_{\Omega}\left|\nabla v_{0}(x)\right|^{2} d x\right)^{(p-m) / m}
\end{aligned}
$$

Now, we assume further that

$$
0<\left\|\nabla w_{0}\right\|^{2 \beta}<\min \left\{\frac{\varepsilon_{0}}{C_{1}},\left(\frac{1}{2 C_{3}}\right)^{(p+m) / 2(p-m)}\right\}
$$

It follows that

$$
\frac{1}{m+1} \frac{d}{d t} \int_{\Omega} v^{1 / m+1} d x \leq-\frac{1}{2} \int_{\Omega}|\nabla v|^{2} d x \leq-\frac{\lambda_{1}}{2} \int_{\Omega} v^{2} d x
$$

Just as in the proof of Theorem 2.5, we see that there exist constants $\mu_{1}, \mu_{2}>0$, such that

$$
\int_{\Omega} v^{(m+1) / m} d x \leq \frac{1}{\left(\mu_{1} t+\mu_{2}\right)^{1 /(\beta-1)}}
$$

This completes the proof.

REMARK 4.3. The result in Theorem 2.6 is invalid for "large" initial data. In fact, from the proof of Theorem 2.6, we see that for some constant $C_{4}>0$,

$$
\int_{\Omega} v(x, t)^{1 / m+1} d x \geq C_{4}>0
$$

provided that $\int_{\Omega} v_{0}(x)^{1 / m+1} d x$ is large enough. 
ACKNOWLEDGEMENT. This paper was supported partially by the grant for the project of the most of China and partially by the NNSF of China (19971004).

\section{REFERENCES}

[1] D. Andreucci and E. di Benedetto, On the Cauchy problem and initial traces for a class of evolution equations with strongly nonlinear sources, Ann. Scuola Norm. Sup. Pisa Cl. Sci. (4) 18 (1991), no. 3, 363-441. MR 92m:35146. Zbl 762.35052.

[2] D. Aronson, M. G. Crandall, and L. A. Peletier, Stabilization of solutions of a degenerate nonlinear diffusion problem, Nonlinear Anal. 6 (1982), no. 10, 1001-1022. MR 84j:35099. Zbl 518.35050.

[3] C. Bandle, M. A. Pozio, and A. Tesei, The asymptotic behavior of the solutions of degenerate parabolic equations, Trans. Amer. Math. Soc. 303 (1987), no. 2, 487-501. MR 88i:35015. Zbl 633.35041.

[4] E. di Benedetto, Continuity of weak solutions to a general porous medium equation, Indiana Univ. Math. J. 32 (1983), no. 1, 83-118. MR 85c:35010. Zbl 526.35042.

[5] V. A. Galaktionov, A boundary value problem for the nonlinear parabolic equation $u_{t}=\Delta u^{\sigma+1}+u^{\beta}$, Differentsial'nye Uravneniya 17 (1981), no. 5, 836-842 (Russian). MR 82m:35074. Zbl 468.35056.

[6] M. E. Gurtin and R. C. MacCamy, On the diffusion of biological populations, Math. Biosci. 33 (1977), no. 1-2, 35-49. MR 58\#33147. Zbl 362.92007.

[7] N. Mizoguchi, Periodic solutions for degenerate diffusion equations, Indiana Univ. Math. J. 44 (1995), no. 2, 413-432. MR 96i:35066. Zbl 842.35049.

[8] M. Nakao, Periodic solutions of some nonlinear degenerate parabolic equations, J. Math. Anal. Appl. 104 (1984), no. 2, 554-567. MR 86d:35013. Zbl 565.35057.

[9] T. Namba, Density-dependent dispersal and spatial distribution of a population, J. Theoret. Biol. 86 (1980), no. 2, 351-363. MR 81i:92024.

[10] M. M. Porzio and V. Vespri, Hölder estimates for local solutions of some doubly nonlinear degenerate parabolic equations, J. Differential Equations 103 (1993), no. 1, 146178. MR 94d:35015. Zbl 796.35089.

[11] P. E. Sacks, Global behavior for a class of nonlinear evolution equations, SIAM J. Math. Anal. 16 (1985), no. 2, 233-250. MR 86f:35031. Zbl 572.35062.

[12] A. Tesei, Time periodic solutions of a degenerate parabolic problem, Actual Problems in Analysis and Mathematical Physics (Rome), Dipartimento di Matematica, Universita di Roma "La Sapienza", 1993, pp. 239-250. MR 94k:35169. Zbl 798.35086.

Yin JingXue: Department of MATHEMATICS, JiLin UniVERSity, ChANGCHUN 130012, ChinA

E-mail address: yjx@mai 1.j1u.edu.cn

WANG Yifu: Department of ApPlied Mathematics, Beijing Institute of Technology, BEIJING 100081, CHINA

E-mail address: wang_yifu@263.net 


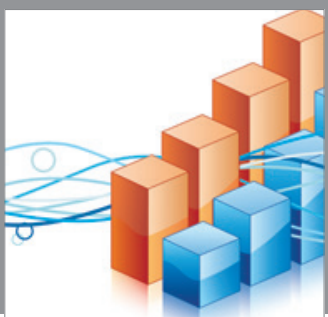

Advances in

Operations Research

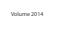

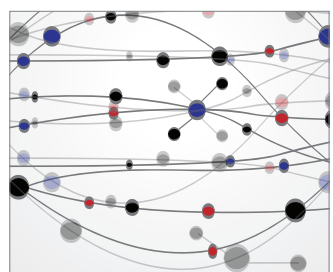

\section{The Scientific} World Journal
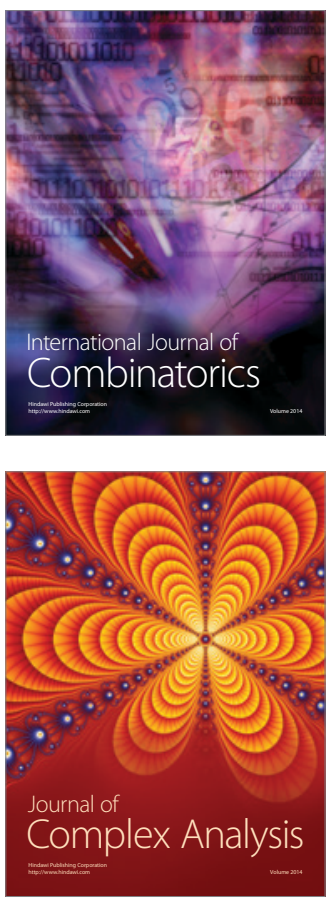

International Journal of

Mathematics and

Mathematical

Sciences
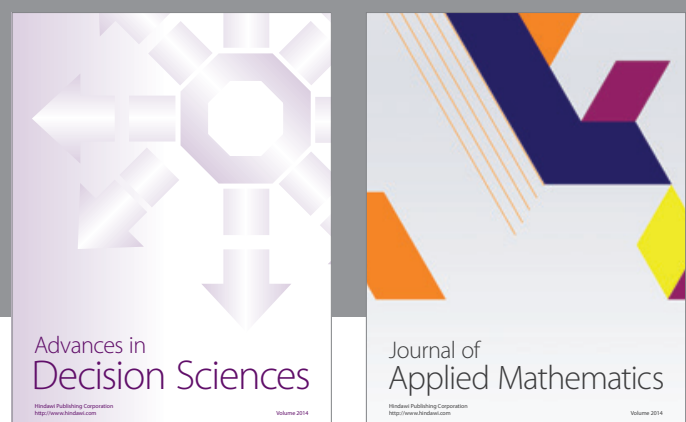

Journal of

Applied Mathematics
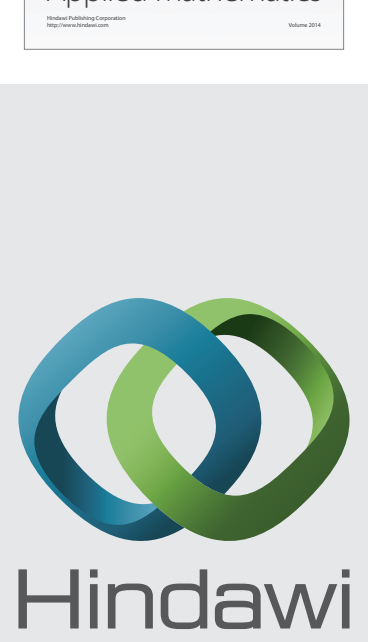

Submit your manuscripts at http://www.hindawi.com
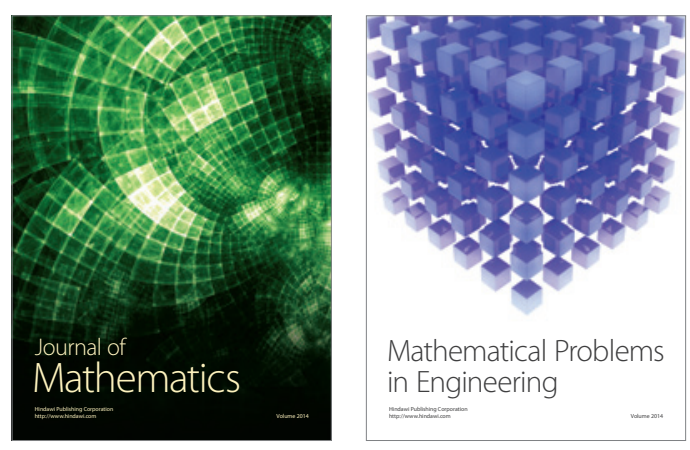

Mathematical Problems in Engineering
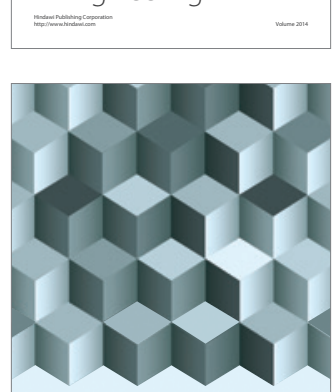

Journal of

Function Spaces
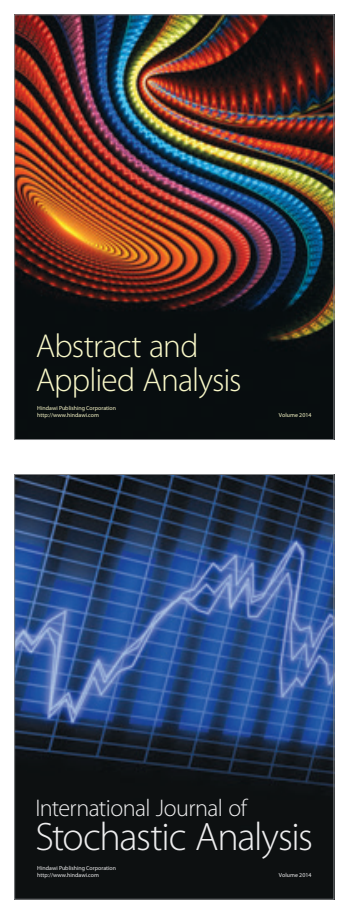

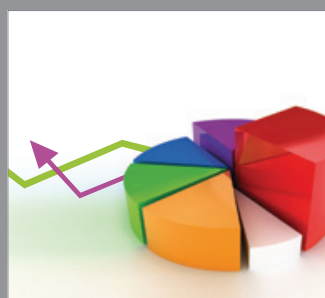

ournal of

Probability and Statistics

Promensencen
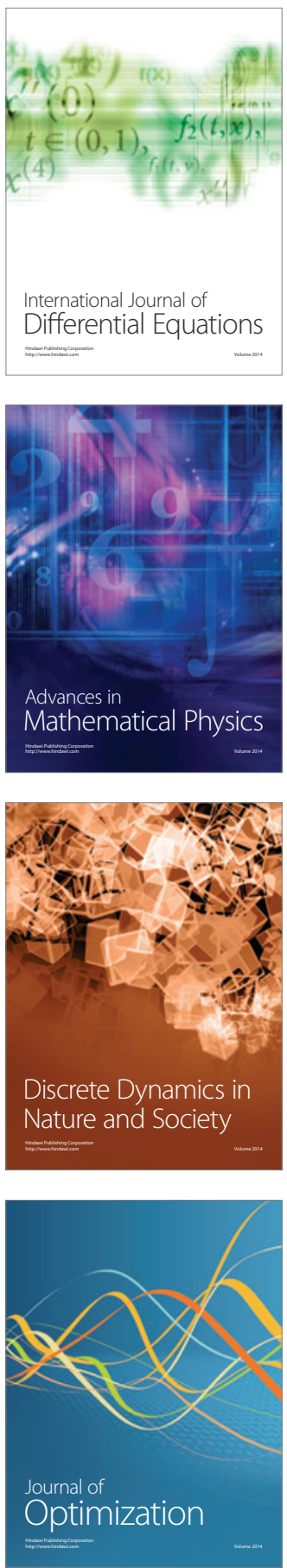